\title{
ENERGY SOURCES OF BLOOD CIRCULATION AND THE MECHANICAL ACTION OF THE HEART*
}

\author{
BY \\ LEON MANTEUFFEL-SZOEGE \\ From the Surgical Department of the Wolski Hospital, Warsaw, Poland
}

(RECEIVEd FOR PUblication AUGUST 12, 1959)

The principle that " the movement of the blood is maintained by the heart which acts as a pump" continues to be binding in modern physiology (Evans, 1952). Certain doubts, however, appear when the power of the heart muscle is regarded as the exclusive or even the main source of energy for the whole cardiovascular system. The question may be asked, Is there no other motive force in the circulatory system, besides the heart muscle, that might be significant ? It is not easy to reconcile the principle of the dominant role of the heart, in the circulation, with the fact of the existence of the venous return energy, but one example from the field of cardiovascular surgery may illustrate that role.

Fig. 1 is an angiogram of a patient with an anastomosis between the superior vena cava and the right pulmonary artery. (The patient was operated on by Dr. Glenn from New Haven, Connecticut, U.S.A.) It was a case of complete transposition of the great vessels with stenosis of the pulmonary artery, and after the operation a great improvement in the patient's condition was observed.

It is difficult to understand the haemodynamics of this part of the patient's circulation, where the systemic venous circulation is connected directly -by-passing the heart-with the arterial pulmonary circulation. How is it possible for the blood, pumped by the heart, impeded by the dense net of capillaries, to cross them and still retain enough energy to pass the second "filter" of the pulmonary capillaries as happened in Dr. Glenn's (1958) case ?

The energy of the venous blood flow can hardly be explained by the small resistance of the capillaries. On the contrary, it seems that the strength of the resistance met by the blood during its passage through the capillaries is underesti-

*This work was supported by a grant from the Polish Academy of Sciences. mated in the teaching of physiology. This problem can be illustrated by the following experiment :

Water from reservoir A (Fig. 2) flows out through tube $B$ with a certain velocity. In the course of tube $B$ is reservoir $C$. If reservoir $C$ is filled (Fig. 3) with a rather tightly packed rubber sponge, the water outflow from tube $\mathrm{C}$ will be diminished many times.

For our experiment, we used reservoir $A$ of 8 litres capacity, the pressure of the water column was about $120 \mathrm{~cm}$., the capacity of reservoir $C$ was 3 litres, and the diameter of tube $B$ was $1.2 \mathrm{~cm}$. ; when a $9 \mathrm{~cm}$. thick rubber sponge was put into reservoir $C$ the flow of the water out of the system was immediately diminished at least six times, depending upon the position of the sponge. The pressure at the tube $\mathrm{C}$ outlet was barely measurable.

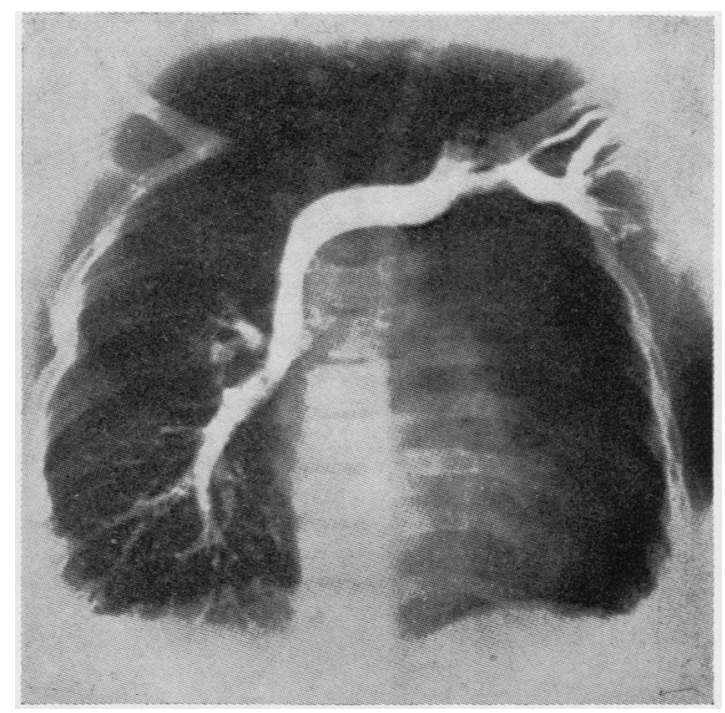

FIG. 1.-Angiography of the patient with anastomosis between the superior vena cava and the right pulmonary artery. (By courtesy of Dr. Glenn.) 


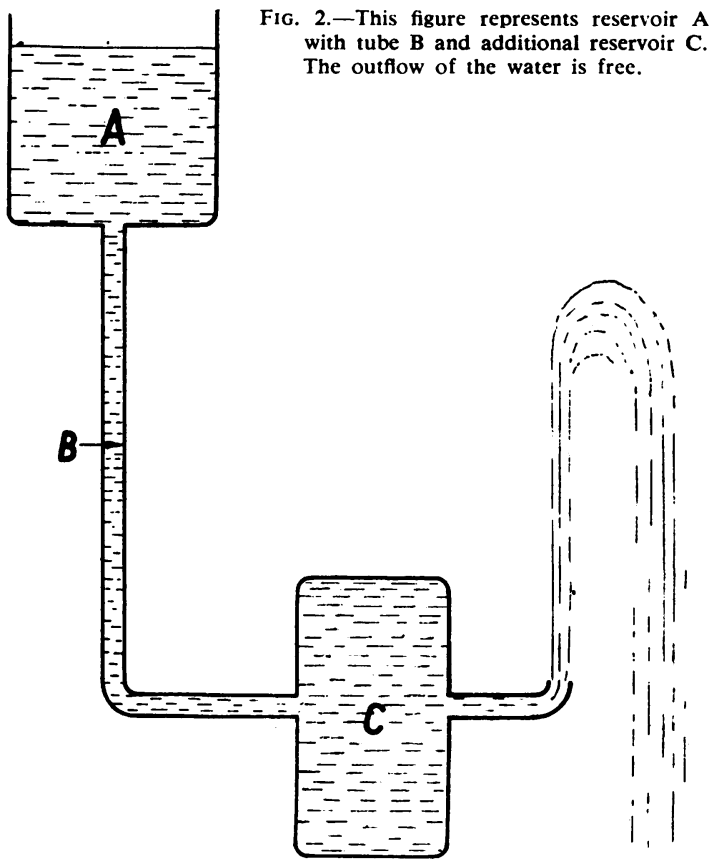

This experiment shows, in a simple analogy, the role of the capillaries as an important source of resistance to the blood flow. The sponge represents the capillaries, with the reservation that, though the resistance in the experiment has some elasticity, it does not have the same characteristics as the capillaries have in a living organism. Consequently one is led to believe that the estimation of the hydrodynamic conditions in the circulation is based on an erroneous assessment of the resistance existing in the system. The resistance is calculated on the basis of the height of the pressure existing in particular parts of the circulatory system as well as by the amount of blood flowing through the system. In this calculation only the work done by the heart is considered and no notice is taken of the possible existence of other, additional, sources of energy in the circulation. It might be supposed that the additional sources are particularly active in the territory of the capillaries, which makes the passage of blood quicker. The action of additional energy would result in a spurious diminishing of the resistance of the circulation, and the vis a tergo of the heart would then have less work to do.

It seems that a much higher pressure would be needed in the circulation of living organisms to move so much blood through the capillaries within a limited time. The efficiency of the circulation

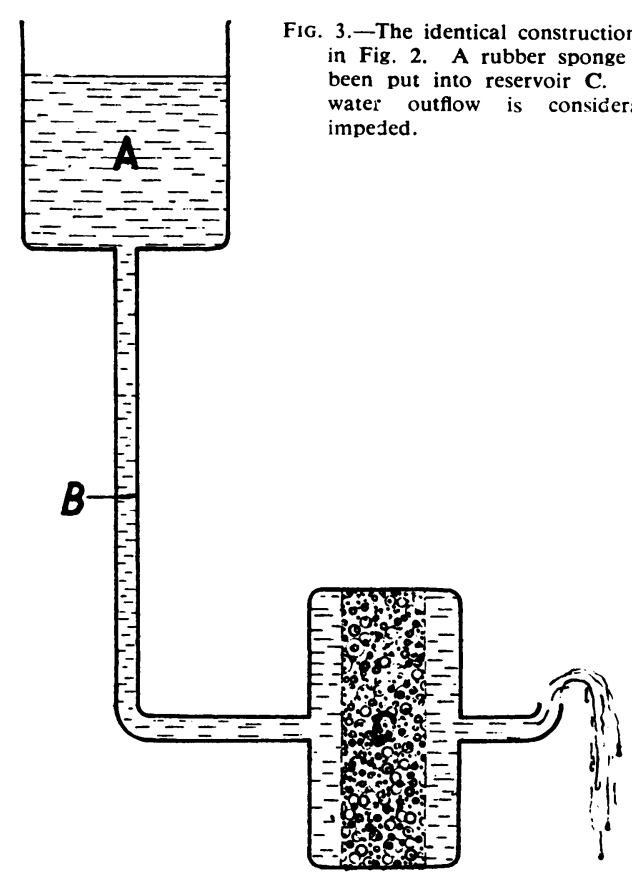

can be so great only because of some additiona source of energy.

In an attempt to clarify these problems, a series of observations on the circulatory systems of 3 릉 and 4-day-old hen embryos (Manteuffel-Szoege Turski, and Grundman, 1959) was made. We ha $\vec{b}$ assumed that the embryonic circulation, which is at that time already well developed, contains the same, if one may say so, essential phenomenon of the circulation as can be found in adults. The embryonic circulatory system has the advantage of being "simplified" and free of a number of mechanical complexities characteristic of the adule organism. It is in a sense a true biologicato "model" of the circulatory system in an adult At the four-day-old stage of development theos lung circulation does not exist; the heario consists of one ventricle and one atrium and in has no valves (Fig. 4). The force of gravitation ${ }_{0}^{\omega}$ need not be taken into account because the embryo. together with its extra-embryonic circulatory? system, is a flat disc lying at one level, and the circulatory system can easily be seen through $a^{+}$ magnifying glass either as a whole or in large fragments. Using a special thermostat we wereop able to observe the embryos at definite temper $-\frac{?}{10}$ atures (Fig. 5).

We established that in hypothermia the venouse system reacts first. The circulation slows down 
and finally stops earlier than in the arterial system. The venous circulation stops completely at a temperature of $20-25^{\circ}$ C. At the same temperature the arterial circulation shows an oscillating but not progressive movement, i.e., after every systole the blood moves forward a little and then returns to the initial point; in other words, at a temperature of $20-25^{\circ} \mathrm{C}$., the blood circulation is interrupted, but the oscillating movement in the main arteries continues. The heart beat usually stops at a temperature lower than $20^{\circ}$ C. The heart rate decreases in direct proportion to the lowering of the temperature. If an embryo is left for from 30 to 90 minutes at a temperature in which the venous circulation has stopped but the heart is still beating, it can be seen that the blood begins to collect in the regions of the peripheral parts of the extra-embryonic arterial system, producing capillary overfilling.

Also, we noticed a number of phenomena in connexion with mechanical manipulations on the embryo. We cut the heart of an embryo from the great vessels, thus producing haemorrhage. The venous circulation or, strictly speaking, the blood movement in the veins during haemorrhage in the conditions of normothermia continues in 3- to 4-day-old embryos for more than 10 or sometimes even for 15 minutes. The fact should be stressed that in these conditions no return spurt is found and there is no haemorrhage from the arteries. There is venous bleeding, whereas in the peripheral arteries the movement of the blood is centrifugal.

The following conclusions suggest themselves: The blood seems to possess another source of energy besides that imparted to it by the heart. This additional energy manifests itself with particular clarity in the venous circulatory system, a conclusion supported by our observations that in hypothermia the venous circulatory system stops first while the heart is still beating. In temperatures of 20 to $25^{\circ} \mathrm{C}$. the action of the heart still produces inefficient, oscillating blood movement in the arteries.

In other words, the hypothesis may be put forward that there exist two different energy moments in blood circulation-one that can be observed in the arterial system, and this is dependent on the action of the heart; and the other, an additional one that manifests itself, above all, in the kinetic energy of the venous circulation system.

The conception that there exists an additional energy moment is also favoured by the fact that the venous circulation continues for a long time

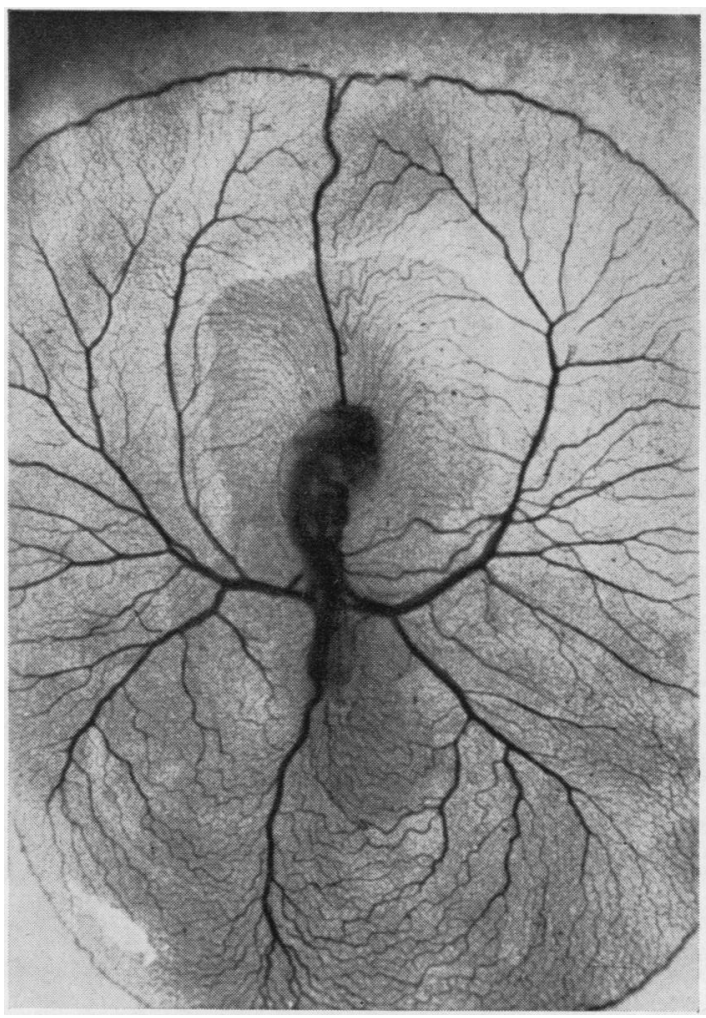

FIG. 4.-A 5-day-old chicken embryo. Note the big extra-embryonic circulation.

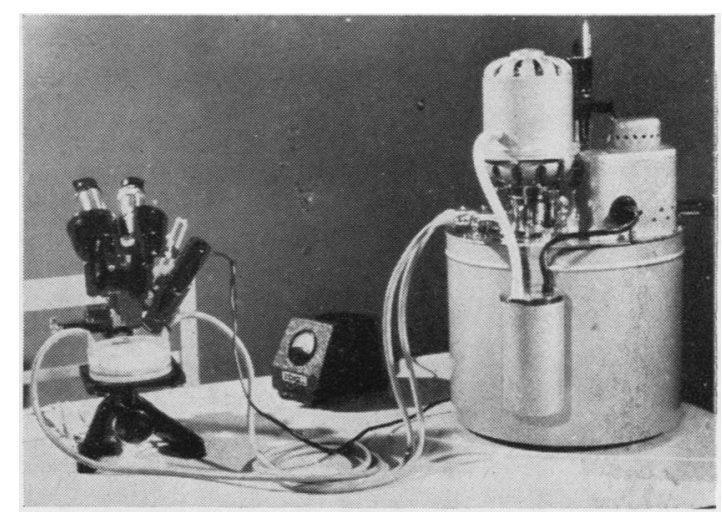

FIG. 5.-Thermostat used for embryonic circulation examinations.

simultaneously with the haemorrhage of an embryo. If the haemorrhage from an embryo after its destruction (cutting out of the heart) were a strictly mechanical problem, it would not last as long as $\mathbf{2 0}$ minutes. 


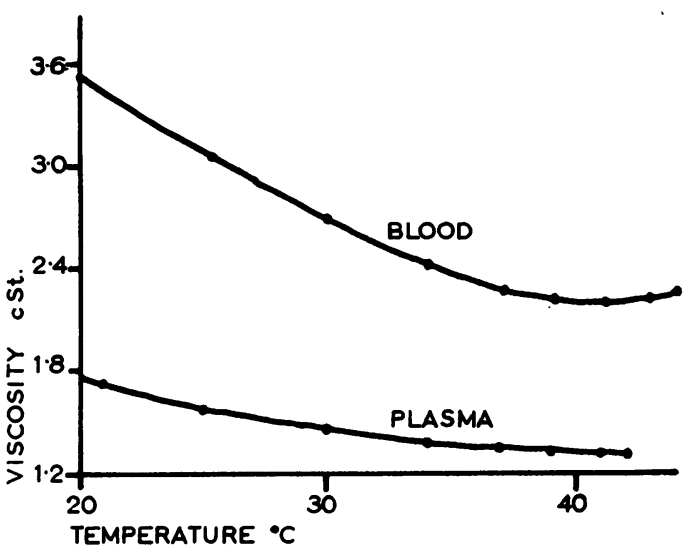

FIG. 6. - Correlation between blood and plasma viscosity and temparature. Top curve represents the viscosity of blood, bottom curve that of plasma.

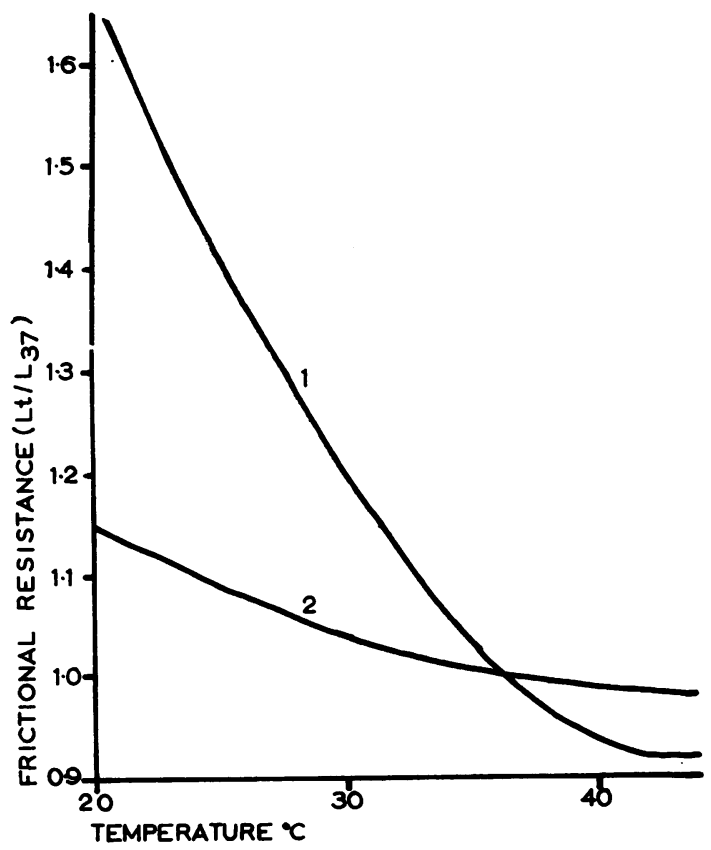

FIG. 7. - Relation between viscosity and friction-work (in formula: logarithm of viscosity-inverse of temperature) regarding temperature up to $37^{\circ} \mathrm{C}$. Curve 1 refers to blood, curve 2 refers to plasma.

It has to be added that during very exact observation of the extra-embryonal circulation, especially the venous and capillary parts of it, no vasomotor motility could be seen that could be considered as an energy factor causing the blood flow. The blood flow seems to be independent of any external factors.
If it is assumed that the conception of the above mentioned additional energy of the circulation if true, the question must inevitably follow, What is the character of this energy? I submit that the circulation is inseparably connected with the thermal conditions of the subject.

It is well known that the circulation, particularly in thermo-stable organisms, can take place only under certain optimum conditions of temperature $\vec{\omega}$ and the thermal conditions for circulation have become particularly conspicuous recently owing tox studies on hypothermia. In connexion with the question of hypothermia I draw the attention of the reader to the fact that the blood viscosity is influenced by lowering of the temperature. To understand the problem better, I carried out, in co-operation with Dr. Sitkowski and $\mathrm{Mr} z$ Maczenski, experiments on the influence of the lowering of temperature on the viscosity of heparinized blood (Manteuffel-Szoege, Sitkowski $\overrightarrow{x o}$ and Maczenski, 1959). The curve in Fig. 6 shows the rise of blood viscosity in relation to the lowering of temperature. The increase of viscosity is so considerable that it influences the action of the circulation. If we assume that the work of the circulation increases in the same $\frac{O}{\mathbb{O}}$ degree as the viscosity, or strictly speaking as the blood rubbing work, it appears that the work of the circulation at $20^{\circ} \mathrm{C}$. compared with that at ${ }^{3}$ $37^{\circ} \mathrm{C}$. increases by about $50 \%$ (Fig. 7). These calculations would be binding if the flow speed? and the amount of passing blood as well as the width of blood vessels were the same at different? temperatures. Thus the thermal factor in the $x$ circulation cannot be reduced to the creation of certain optimum conditions for this system. The thermal factor is of direct dynamic significance, $\frac{O}{3}$ influencing the energy balance of the circulation. If we reverse the influence of the temperature ons viscosity we can state that increased temperature, by diminishing viscosity, reduces resistance ande. consequently also the work of the circulation. OfN the internal organs it is the liver that shows the highest temperature-higher than that of the hearto -and the liver is the most dynamic centre of thew venous circulation (Horvath, Rubin, and Foltz? 1950).

Thus the energy factor of the circulation is strictly connected with the thermal moment.? Nevertheless, on the basis of the presented evidence only, heat itself, though an indispensable

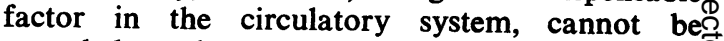
regarded as the source of the additional energy $\mathbb{0}$ of the circulation.

The assumption that there exists, besides the work of the heart, the additional energy in theo 


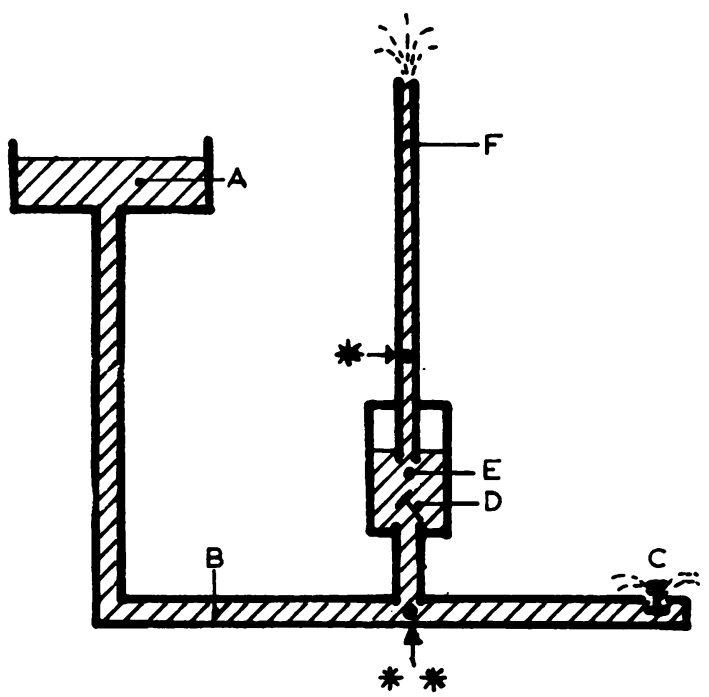

FIG. 8.-Diagram of a water ram, as constructed by the author. With one asterisk the place is marked where the catheter was introduced into the analogue of the great artery. Two asterisks mark the point of introduction of the catheter into the analogue of the ventricle.

circulation forces us to reconsider the present opinions as to the nature of the mechanical action of the heart.

The heart action is compared to that of a pump. Is it really true that the heart works like a pump ? A pump sucks in fluid from a reservoir, which is a hydrostatic system and not a hydrodynamic one. In the circulation, on the other hand, not only is blood ejected from the heart but it flows into the heart. The heart is a mechanism inserted into the blood circuit, and so it is a very peculiar kind of pump.

Blood flowing into the ventricles under low pressure leaves them under much higher pressure. The question could arise whether there exists in hydraulics an instrument that, when inserted into the blood circulation, would change its pressure from a lower to a higher one. An instrument of this kind exists and it is called the water ram. The idea of the heart working on a principle similar to that of the water ram has already been put forward (Schmid, 1892 ; Steiner, 1920; Havlicek, 1937), and I have elaborated the analogy between the heart action and that of the hydraulic ram in co-operation with Dr. T. Gonta (ManteuffelSzoege and Gonta, 1958).

I should like to present briefly the principle of the work of the water ram (Fig. 8) set out before in a paper on the principle of the heart's action. Of basic importance in this device is the so-called "water hammer." By this is understood the rapid change of kinetic energy, existing in running water, into the potential energy of pressure built up in the water when the flow is abruptly stopped. The pressure can rise considerably and can be many times higher than the pressure prevailing in the liquid before it was stopped.

The water (Fig. 8) from reservoir A flows down through tube $B$ and reaches its end part, where there is an opening equipped with valve $C$. This valve, by virtue of its weight, exerts some resistance to the outflowing water, but is finally lifted by the current and obstructs the opening. The sudden halt in the water current causes a violent rise of pressure in the system, i.e., the previously described phenomenon of the water hammer occurs. The rising pressure opens valve $D$, through which water flows into cylinder $E$, and from there to the outflow tube $F$. When the water in this tube rises to the level of the reservoir A, water surface, valve $D$ is shut by the weight of the water column above it. It becomes impossible for the water, trapped in tube $F$, to flow back into tube $B$.

The pressure in tube $B$, lowered again by the spurt through the chamber $E$ and tube $F$, is not able to keep the valve $C$ in the shut position any longer. The valve $\mathrm{C}$, by the force of gravity, falls down and the cycle described is started again.

When this happens, the air contained in chamber $E$ is compressed, then it expands and forces some of the water from chamber $E$ into outflow tube $F$. As soon as the pressures of the water and air become equal, the action described above starts again automatically. The presence of air in chamber $\mathbf{E}$ is not indispensable for the water ram action. In order to put the water ram into operation it is necessary to push valve $\mathrm{C}$ several times from above downwards; the device then starts to work automatically as long as there is water in the reservoir $A$. It is worth mentioning that the outflow valve $C$ can be situated between the reservoir and chamber $E$ as well as at the end of tube $B$.

The analogy between the heart and the water ram is as follows. The heart is, as maintained also by Havlicek (1937), a combination of two synchronously working water rams corresponding to the right and the left heart. Tube $F$ represents the aorta or the pulmonary artery with valve $D$ in lieu of the aortic or pulmonary artery valves. The part of the system under valve $D$ can be considered an analogue of the ventricle. It is difficult to say just how far in the direction of reservoir $A$ the analogy is valid. Outflow valve $\mathrm{C}$ partly plays the role of the bicuspid or tricuspid valve. The correspondence is only partial. It may be difficult to understand this analogy, the difficulties being 


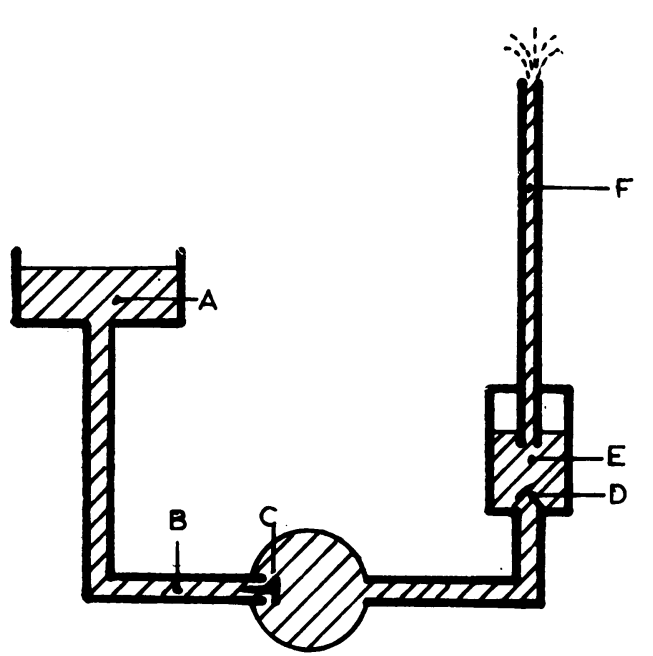

FIG. 9.-The diagram of a water ram constructed in a way more resembling the construction of the heart. Such a water ram, without a biological pacemaker, cannot work automatically.

anatomical, but if the problem is approached from the physiological point of view it becomes easier to understand. Outflow valve $C$ is of crucial importance in the construction of the water ram. This valve opens and closes rhythmically during the ram's operation. The rapid, violent closing of the valve determines the most essential function of the device, i.e., the sudden rise of pressure near the valve; this increase of pressure causes valve $D$ to open and the water to surge into outflow tube $F$ above the reservoir $\mathrm{A}$ water surface level. The same thing happens in the heart. Blood flows into the ventricle at the moment when the bicuspid or tricuspid valve closes, the stream of blood is abruptly stopped and the blood wave rebounds from the closed valve and from the heart muscle which becomes stretched.

Owing to the rebounding of blood from the so-called elastic elements such as the valve and the muscle the pressure suddenly rises in the ventricle and consequently also in the aorta and the pulmonary arteries. Thus the principle of the water ram action can be applied to the heart's work. The objection might be raised that in the blood circulation there is no place for blood loss analogous to the water loss in the water ram operation. But the water ram is only a mechanical instrument.

In order to make the comparison between the water ram and the heart easier to imagine, a special model (Fig. 9) can be constructed. It would not act automatically, but it would serve the purpose of understanding the analogy between the two systems. The outflow valve $\mathrm{C}$ has been closed. An instrument of this type cannot work automatically without a suitable biological pace은 maker. The muscle of the ventricles in the heare constitutes such a pacemaker and the energy? produced by the muscle corresponds to the energya generated in the hydraulic ram by the loss of water through valve $C$.

The analysis of the mechanical action of the heart presented here explains this action on the $\vec{\omega}$ principle of the water ram. A more detailed study of this principle will bring more arguments in $\vec{x}$ favour of my thesis. One argument is the observa $\frac{\vec{c}}{\mathrm{r}}$ tion made by Schmid (1892) that the apex beat precedes the ejection of blood from the ventricles and not vice versa. With every stroke the pumpo ejects all the liquid it contains. The heart, on the other hand, does not empty itself entirely during systole: one-third of the blood contained in then heart during diastole remains there while two $\widehat{\widehat{S}}$ thirds are expelled into the arteries (Heilmeyer, 1956). This observation favours the water ramo theory rather than that of the sucking and forcing pump.

The last argument I shall quote consists in thes striking similarity between the tracing of the bloodo pressure obtained from the ventricles of the hearto and from the great arteries and the tracing of pressures taken from the respective parts of the water ram model (Manteuffel-Szoege and Gonta 1958). The tracing shown in the upper part of Fig. 10 represents the changes of pressure in theo water ram near the rhythmically working outflow valve of the ventricle analogue. The place whereo the catheter was placed while the tracing was

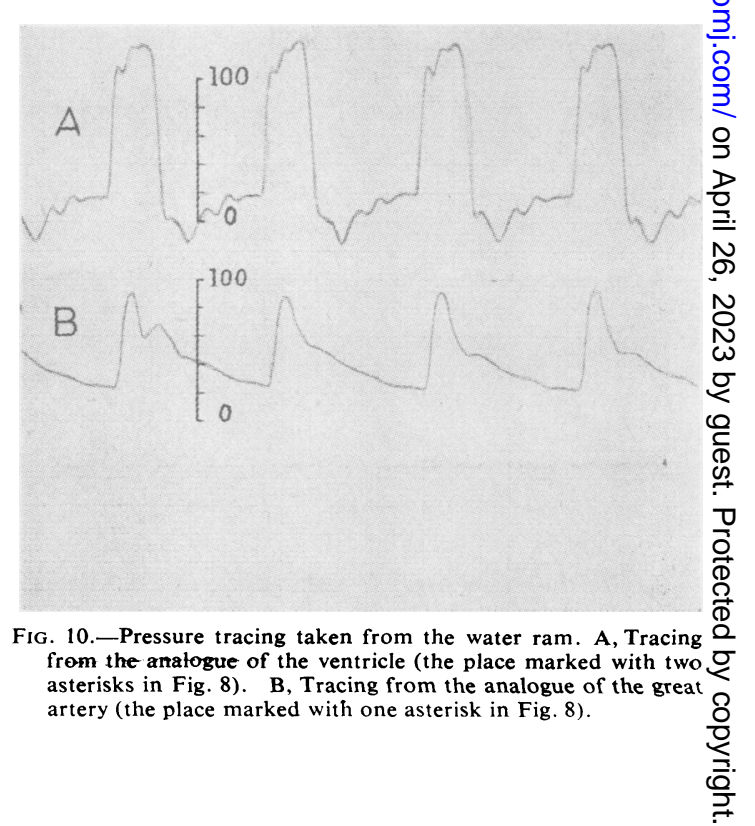




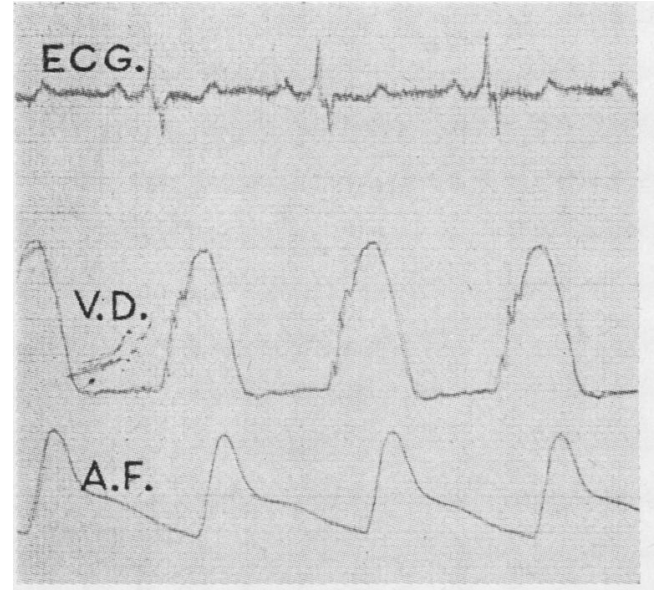

FIG. 11.-V.D., Tracing of the pressures in the right ventricle of a man, taken during heart catheterization. A.F., Tracing of the pressure in the femoral artery, taken by direct puncture in the same man.

recorded is marked in Fig. 8 by two asterisks. This tracing is almost identical with the curve illustrating the pressures in the right ventricle registered during cardiac catheterization of an 18year-old man (Fig. 11). The lower tracing (Fig. 10) comes from the tube corresponding to the pulmonary artery or aorta. The position of the catheter is marked in Fig. 8 with one asterisk, and the record resembles very much that of the major artery (Fig. 11).

The comparison of the tracing obtained from the water ram with that from corresponding places in the human circulatory system shows that the hydrodynamic phenomena in both systems are almost identical.

The work of the heart, as I have interpreted it, would not play a unique role, in the energy sense, in relation to the circulation; a very important role would be ascribed to the previously mentioned additional energy factor of the circulation.

\section{SUMmary}

I believe that the observations I have put forward tavour the conception that there exists, besides the work of the heart, another, additional, source of circulatory energy. The additional energy of the circulation manifests itself most distinctly in the venous system and is strictly connected, as is the whole circulatory action, with the temperature in various organs.

It may be necessary to modify the present interpretation of the mechanical work of the heart as a pump and compare it to the hydraulic instrument called the water ram.

\section{REFERENCES}

Evans, C. L. (1952). Principles of Human Physiology, 11th ed. Churchill, London.

Glenn, W. W. L. (1958). New Engl. J. Med., 259, 117.

Havlicek, H. (1937). Arch. Kreisl.-Forsch., 1, 188.

Heilmeyer, L.(1956). Vorträge aus dem Gebiet der klinischen Chemic und Cardiologie. Thieme, Stuttgart.

Horvath, S. M., Rubin, A., and Foltz, E. L. (1950). Amer. J. Physiol., 161, 316.

Manteuffel-Szoege, L., and Gonta, T. (1958). Minerva cardioangiologica Europea, 6, No. 2, p. 261.

Sitkowski, W., Jr., and Maczenski, S. (1959). Ibid., 7, No. 3, p. 256

Turski, Cz., and Grundman, J. (1959). About the Energy Sources of the Blood Circulation. In the press.

Schmid, K. (1892). Wien. med. Wschr., 42, 578, 622, 662.

Steiner, R. (1920). Vortrags-Zyklus für Aertzte und Medizinstudierende. Der kommende Tag, Stuttgart. 